\title{
Local Signs and Symptoms in Spontaneous Cervical Artery Dissection: A Single Centre Cohort Study
}

\author{
Lukas Mayer, ${ }^{\text {a }}$ Christian Boehme, ${ }^{\mathrm{a}}$ Thomas Toell, ${ }^{\mathrm{a}}$ Benjamin Dejakum, ${ }^{\mathrm{b}}$ Johann Willeit, ${ }^{\mathrm{a}}$ \\ Christoph Schmidauer, ${ }^{\mathrm{a}}$ Klaus Berek, ${ }^{\mathrm{c}}$ Christian Siedentopf, ${ }^{\mathrm{d}}$ Elke Ruth Gizewski, ${ }^{\mathrm{d}}$ Gudrun Ratzinger, \\ Stefan Kiechl, ${ }^{a}$ Michael Knoflach ${ }^{\mathrm{a}}$ \\ aDepartment of Neurology, Medical University Innsbruck, Innsbruck, Austria \\ ${ }^{b}$ Department of Neurology, Unfallklinik Murnau, Murnau am Staffelsee, Germany \\ 'Department of Neurology, Bezirkskrankenhaus Kufstein, Kufstein, Austria \\ ${ }^{d}$ Department of Neuroradiology, Medical University Innsbruck, Innsbruck, Austria \\ eDepartment of Dermatology, Medical University Innsbruck, Innsbruck, Austria
}

\section{Dear Sir:}

Spontaneous cervical artery dissection (SCAD) is one of the main causes of ischemic stroke in young adults. ' Local symptoms (LSs) are common in SCAD and often predate ischemic events, yet little is known about their frequency and prognosis. ${ }^{2-9}$

Consecutive patients with SCAD admitted to the Medical University of Innsbruck between July 1996 and January 2017 were included in this study. The diagnosis of SCAD was obligatorily confirmed by magnetic resonance imaging (MRI) documentation of the intramural hematoma in $\mathrm{T}_{1}$-weighted fat-saturated sequences. Cases of CAD that occurred spontaneously or following minimal trauma, for example hyperextension, rotation or lateroversion of the neck, were eligible for our study. Patients with high impact trauma with signs of external or internal injury other than $C A D$, and patients with intracranial artery dissection were excluded from this analysis. The electronic medical records of all eligible patients, including discharge, imaging, and outpatient reports, were carefully reviewed with a focus on the frequency, presentation, duration, and long term outcome of LSs. Clinical evaluation of the data was done by an experienced stroke physician. Head/neck pain, Horner's syndrome, lower cranial nerve palsy (IX through XII, isolated or combined), and tinnitus, caused by local mass effect of the dissected vessel on the surrounding tissue, were considered as LSs. All SCAD patients were invited to an extensive standardized follow-up visit within our ReSect-Study, a single centre cohort study investigating possible risk factors for recurrent SCAD. The study was approved by local ethics committee and all participants signed appropriate consent forms.

A total of 259 patients with MRI-confirmed SCAD were enrolled. The median follow-up was 6.0 years (interquartile range [IQR], 6.0; minimum 0.8 to maximum 20.3). No in person follow-up or follow-up records were available in only five patients. In our cohort, males predominated (59.8\%). The median age at onset was 44.4 years (IOR, 14.8; minimum 21 to maximum 87 ). At symptom onset, men were older than women (median, 47.4 years $[I Q R, 14.0]$ and $40.5[I O R, 13.1], P<0.001)$. A total of 190 subjects of our cohort suffered ischemic stroke/transient ischemic attack (73.4\%) (Table 1).

LSs were evident in 212 of the 259 SCAD patients (81.9\%) with head/neck pain being the most frequent ( $n=205,79.2 \%)$, followed by Horner's syndrome $(n=42,16.2 \%)$, tinnitus ( $n=19$, $7.3 \%)$, and lower cranial nerve palsy $(n=13,5.0 \%)$. Multiple LSs were seen in 61 of 259 patients (23.6\%). Headache was the only LS that was present in all patients with multiple LSs and head/ neck pain combined with Horner's syndrome was the most common combination of symptoms (34 of $61,55.7 \%$ ). In all of the cases with cranial nerve palsy, an expansive pseudo-aneurysm was evident. None of our patients had complaints suggestive of cervical nerve root compression. Head/neck pain relieved within a median of 13.5 days $(I Q R, 12)$ and all patients with cranial nerve palsy or tinnitus had full spontaneous remission (maximum duration 5 and 3 months, respectively). Horner's syndrome 
Table 1. Baseline demographics of the full cohort $(n=259)$

\begin{tabular}{|c|c|}
\hline Demographic & Value $^{*}$ \\
\hline Male sex & $155(59.8)$ \\
\hline Age (yr) & $44.4(29.6-59.2)^{+}$ \\
\hline Years of follow-up & $6(0-12)^{+}$ \\
\hline Follow-up visits & $5(0-12)^{+}$ \\
\hline \multicolumn{2}{|l|}{ Characteristic } \\
\hline \multicolumn{2}{|l|}{ Localisation } \\
\hline Anterior circulation & $117(45.2)$ \\
\hline Posterior circulation & $133(51.3)$ \\
\hline Both & $9(3.5)$ \\
\hline Multiple dissections & $40(15.4)$ \\
\hline \multicolumn{2}{|l|}{ Clinical presentation } \\
\hline No ischemia & $69(26.6)$ \\
\hline Stroke/TIA & $190(73.4)$ \\
\hline Local signs and symptoms & $212(81.9)$ \\
\hline Head-/neck pain & $205(79.2)$ \\
\hline Horner's syndrome & $42(16.2)$ \\
\hline Cranial nerve palsy & $13(5.0)$ \\
\hline Tinnitus & $19(7.3)$ \\
\hline \multicolumn{2}{|l|}{ Symptom onset } \\
\hline Local first & $181(69.9)$ \\
\hline Stroke/TIA first & $77(29.7)$ \\
\hline Asymptomatic & $1(0.4)$ \\
\hline \multicolumn{2}{|l|}{ Primary reason for seeking medical help } \\
\hline Symptoms due to ischemic stroke/TIA & $172(66.4)$ \\
\hline Local signs and symptoms & $85(32.8)$ \\
\hline Incidental finding & $2(0.8)$ \\
\hline
\end{tabular}

Values are presented as number (\%) or median (interquartile range).

TIA, transient ischemic attack.

${ }^{*}$ Analysis of categorial variables was done by means of the Pearson chi-square test; ${ }^{+}$Analysis of continuous variables was done by means of the Mann-Whitney U test.

resolved in three-quarters of cases (28 of 37 ) (Figure 1). There was no significant difference in age, sex, vessel segment involvement, or presence of cerebral ischemia between patients with or without full recovery of Horner's syndrome. Furthermore, acute medical therapy and secondary prevention after sCAD had no influence on LS-outcome.

Additional 16 and 12 subjects had Horner's syndrome or lower cranial nerve palsy caused by brainstem ischemia, and none of these patients showed full remission. Head/neck pain was more frequent in vertebral (74.8\%) than in internal carotid artery dissection (74.8\%), and more common in vertebral artery dissection involving proximal V0-V2 segments (94.1\%) than distal V3-V4 segments (82.4\%).

Horner's syndrome and cranial nerve palsy exclusively manifested in patients with internal carotid artery dissection (unless caused by brainstem infarction). Tinnitus was always pulsatile and present in 14 subjects with internal carotid artery dissection (mainly C3 segment) and two with a bilateral dissection of the vertebral arteries (V3 and V4 segments) (Figure 2).

Our comprehensive analysis of a large consecutive cohort of well characterized patients with SCAD indicates that LSs occur frequently. Knowledge of these symptoms could aid in diagnosing (or suspecting) of CAD early in the acute setting, before cerebral ischemia has occurred. In addition, LSs might help to pinpoint the vessel or even vessel segments involved. Frequency and course of LSs in SCAD patients have so far attracted little attention in the literature. ${ }^{2-9}$

To the best of our knowledge this is the first systematic evaluation of the outcome of LSs in SCAD. Our data are relevant to both clinicians and patients as LSs caused by SCAD 


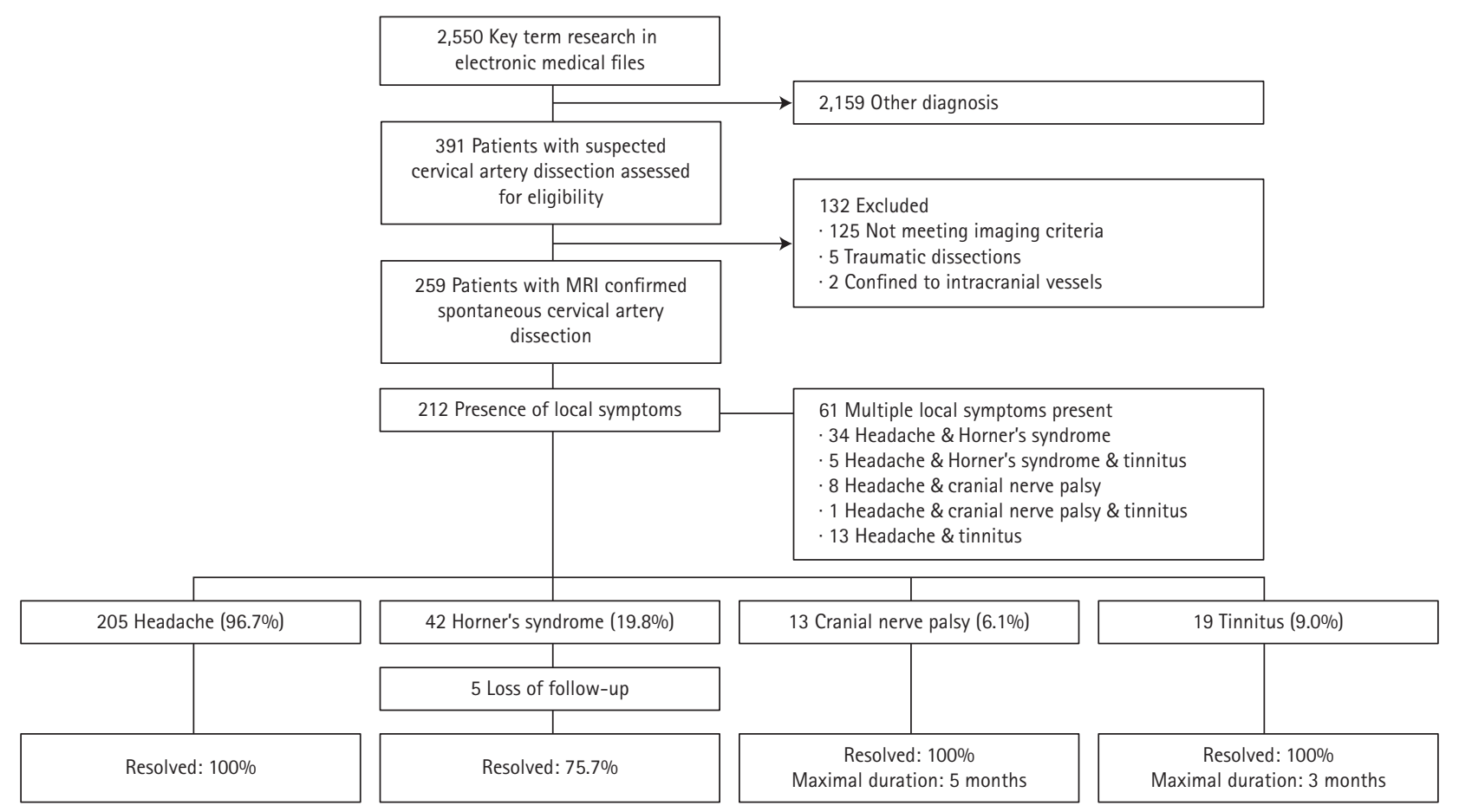

Figure 1. Flow chart of screening, enrolment and prognosis of local symptoms in patients with spontaneous cervical artery dissection ( $n=259)$. In 61 patients, multiple local symptoms were present. MRI, magnetic resonance imaging.

\begin{tabular}{lc}
\hline Vertebral artery & $\%$ V0-V4 $(\mathrm{n}=141)$ \\
\hline Headache & $84.4(119)$ \\
Horner's & 0.0 \\
Cranial nerve palsy & 0.0 \\
Tinnitus & $3.5(5)$ \\
\hline
\end{tabular}

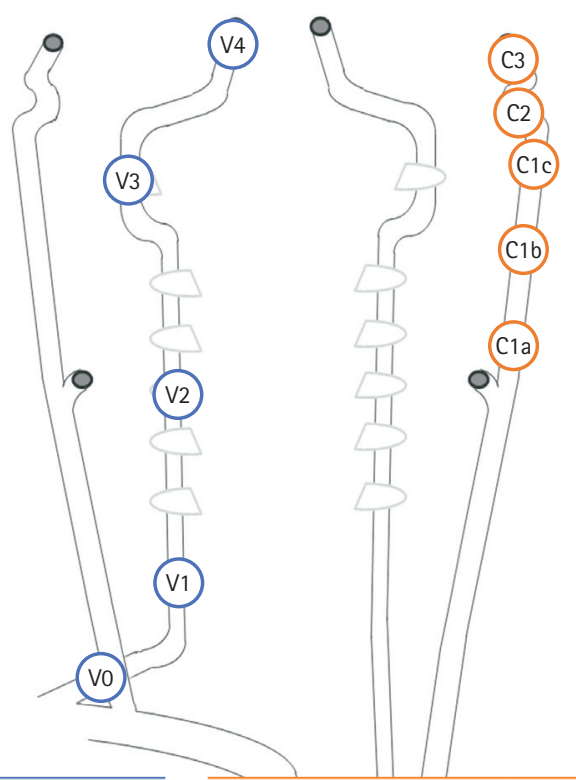

\begin{tabular}{lc}
\hline Internal carotid artery & $\begin{array}{c}\% \\
\text { C1a-C3 }(n=127)\end{array}$ \\
\hline Headache & $74.8(95)$ \\
Horner's & $33.1(42)$ \\
Cranial nerve palsy & $10.2(13)$ \\
Tinnitus & $13.4(17)$ \\
\hline
\end{tabular}

\begin{tabular}{lcc}
\hline Vertebral artery & $\%$ & $\%$ \\
& V0-V2 $(n=51)$ & V3-V4 $(n=119)$ \\
\hline Headache & $94.1(48)$ & $82.4(98)$ \\
Horner's & 0.0 & 0.0 \\
Cranial nerve palsy & 0.0 & 0.0 \\
Tinnitus & $3.9(2)$ & $2.5(3)$ \\
\hline
\end{tabular}

\begin{tabular}{lccc}
\hline Internal carotid artery & $\begin{array}{c}\% \\
\mathrm{C} 1 \mathrm{a}(\mathrm{n}=23)\end{array}$ & $\begin{array}{c}\% \\
\mathrm{C} 1 \mathrm{~b}-\mathrm{C} 2(\mathrm{n}=118)\end{array}$ & $\mathrm{C} 3(\mathrm{n}=5)$ \\
\hline Headache & $69.9(16)$ & $74.6(88)$ & $80.0(4)$ \\
Horner's & $30.4(7)$ & $33.9(40)$ & $20.0(1)$ \\
Cranial nerve palsy & 0.0 & $11.0(13)$ & 0.0 \\
Tinnitus & $21.7(5)$ & $11.9(14)$ & $40.0(2)$ \\
\hline
\end{tabular}

Figure 2. Frequency of local symptoms according to vessel segment involvement in patients with spontaneous cervical artery dissection (sCAD). Patients with simultaneous anterior and posterior circulation $\operatorname{SCAD}(n=9)$ were excluded from this figure. In some patients, a singular dissection involved multiple vessel segments. 
showed excellent mid- to long-term outcome in contrast to LS-mimics such as Horner's syndrome or cranial nerve palsies caused by brainstem ischemia. In detail, all patients with lower cranial nerve palsy caused by compression due to expansive pseudo-aneurysms, showed complete mid-term symptom remission without endovascular repair. Favorable outcome extends to head-/neck pain, the most common LS, which resolved in all patients within a median of 13.5 days and to Horner's syndrome and tinnitus (Figure 1). In contrast, none of the patients with Horner's syndrome or lower cranial nerve palsies caused by brainstem stroke showed full recovery.

In summary, our findings assist physicians in counseling patients about the favorable prognosis of LSs in SCAD and help avoiding unnecessary interventions like endovascular repair of large cervical pseudo-aneurysms.

\section{References}

1. Debette $S$, Leys D. Cervical-artery dissections: predisposing factors, diagnosis, and outcome. Lancet Neurol 2009;8:668-678.

2. Dziewas $R$, Konrad C, Dräger $B$, Evers $S$, Besselmann $M$, Lüdemann $P$, et al. Cervical artery dissection: clinical features, risk factors, therapy and outcome in 126 patients. J Neurol 2003; 250:1179-1184.

3. Baumgartner RW, Arnold M, Baumgartner I, Mosso M, Gön-

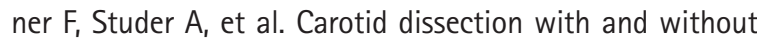
ischemic events: local symptoms and cerebral artery findings. Neurology 2001;57:827-832.

4. Béjot $Y$, Aboa-Eboulé $C$, Debette $S$, Pezzini $A$, Tatlisumak $T$, Engelter $S$, et al. Characteristics and outcomes of patients with multiple cervical artery dissection. Stroke 2014;45:37-41.

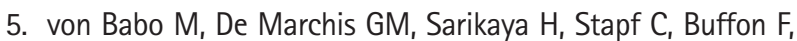
Fischer $\mathrm{U}$, et al. Differences and similarities between spontaneous dissections of the internal carotid artery and the vertebral artery. Stroke 2013;44:1537-1542.

6. Arnold M, Cumurciuc R, Stapf $C$, Favrole P, Berthet $K$, Bousser MG. Pain as the only symptom of cervical artery dissection. J Neurol Neurosurg Psychiatry 2006;77:1021-1024.

7. Traenka C, Dougoud D, Simonetti BG, Metso TM, Debette $S$, Pezzini $A$, et al. Cervical artery dissection in patients $\geq 60$ years: often painless, few mechanical triggers. Neurology 2017;88:1313-1320.

8. Arnold M, Kappeler L, Georgiadis D, Berthet K, Keserue B, Bousser MG, et al. Gender differences in spontaneous cervical artery dissection. Neurology 2006;67:1050-1052.

9. Biousse V, D'Anglejan-Chatillon J, Touboul PJ, Amarenco P, Bousser MG. Time course of symptoms in extracranial carotid artery dissections. A series of 80 patients. Stroke 1995;26:235239.

Correspondence: Michael Knoflach

Department of Neurology, Medical University Innsbruck, A-6020 Innsbruck, Anichstraße 35, Austria

Tel: +43-512-504-81697

Fax: +43-512-504-67-81697

E-mail: Michael.knoflach@i-med.ac.at

Received: October 25, 2018

Revised: December 26, 2018

Accepted: December 26, 2018

This analysis is part by the "ReSect-Study" funded by the OeNB Anniversary Fund (\#15644). Stefan Kiechl, Johann Willeit, and Michael Knoflach are supported by the excellence initiative (Competence Centers for Excellent Technologies [COMET]) of the Austrian Research Promotion Agency FFG: "Research Center of Excellence in Vascular Ageing: Tyrol, VASCage" (K-Project No. 843536) funded by BMVIT, BMWFW, Wirtschaftsagentur Wien, and Standortagentur Tirol. 